\section{Ingeniería}

CONTENIDO

Artículos

Análisis numérico de un sistema de aire acondicionado empleando
mallas estructuradas y no estructurades A

Funciones de regresión para caudales extremos en la Vertiente Rojas, Nazareth; Aguilar, José Francisco; Solís, Hernán. Evaluación de daños por agresión ambiental en viviendas
de concreto reforzado. Solís, Rómel; Moreno, Eric; Jiménez, Felipe; Rosas, Victorino.

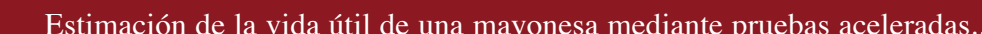
Estimación de la vida útil de una mayonesa
García, Claudia; Molina, Manuel.

Inestabilidad oscilatoria de tensión debido a los motores de inducción …….....................65-75 rde, Gustavo; Araya, Eddie.

Estabilidad y colapso de tensión en sistemas eléctricos Araya, Eddie.

Obtención de pulpa a la soda antraquinona de Tectona grandis creciendo en Costa Rica...........................

Protocolo evaluador para el manejo de desechos y la seguridad ocupacional
aplicado al laboratorio clínico del Hospital San Francisco de Asís de Grecia. Ruiz, Francisco.

9. Euler: su contex
Vargas, Celso.

10. Reserva de resistencia de edificios porticados de concreto armado disenaados conforme al ACI-318/ IBC 2006 ........ Análisis y comentarios

Herrera, Rodolfo.

breve semblanza.

Trabajos de Graduación 2007

Lista de proyectos de graduación de grado y posgrado

Normas

$\sum_{\text {EDTORAL }}$

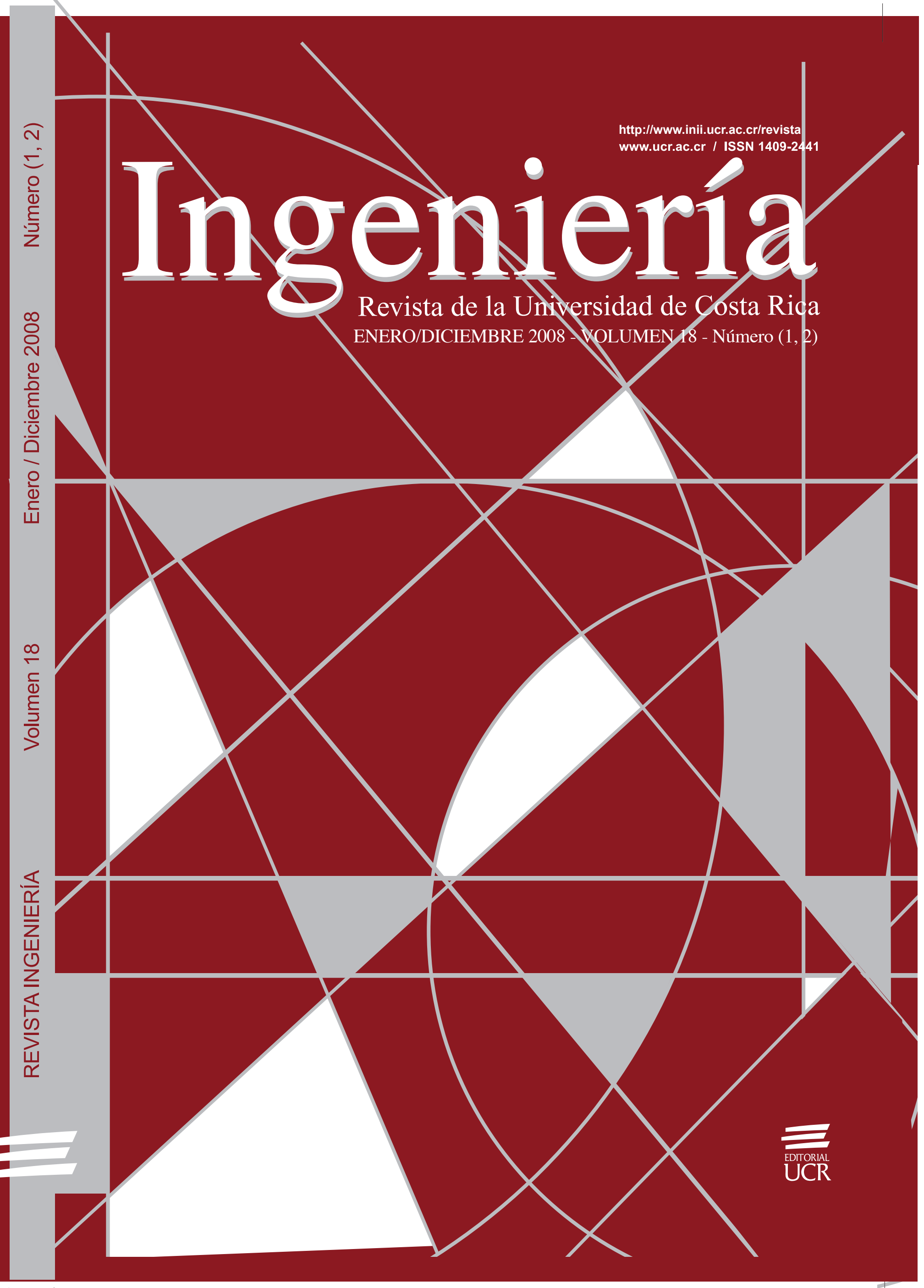




\title{
ESTIMACIÓN DE LA VIDA ÚTIL DE UNA MAYONESA MEDIANTE PRUEBAS ACELERADAS
}

\author{
Claudia García Baldizón \\ Manuel Enrique Molina Córdoba
}

\begin{abstract}
Resumen
La vida útil de una mayonesa se estimó mediante pruebas aceleradas, empleando el índice de peróxidos como indicador de deterioro. El producto se almacenó a $21^{\circ} \mathrm{C}, 35^{\circ} \mathrm{C}$ y $45^{\circ} \mathrm{C}$ durante $210 \mathrm{~d}, 90 \mathrm{~d}$ y $42 \mathrm{~d}$, respectivamente. Se realizaron como mínimo seis muestreos para cada temperatura y los resultados obtenidos se utilizaron para definir la cinética de esta reacción de deterioro. La cinética de la reacción fue de orden cero y las constantes cinéticas encontradas fueron $(0,31,0,173$ y 0,365$) \mathrm{meqO}_{2} / \mathrm{kg} \cdot \mathrm{d}$, en orden creciente de temperatura. Con estas constantes específicas de reacción y el modelo de Arrhenius se obtuvo un valor de energía de activación de $80960 \mathrm{~J} / \mathrm{mol}$, la cual se encuentra dentro del ámbito de las reacciones de oxidación de lípidos. Y por último, se obtuvo una relación para estimar la vida útil de la mayonesa, cuya ecuación general es Vida útil $=10^{(2,907-0,036 \cdot T)}$
\end{abstract}

Palabras clave: mayonesa, vida útil, cinética, oxidación de lípidos.

\begin{abstract}
Was estimated the shelf life of a mayonnaise using accelerated temperatures. The deterioration variable used was the peroxide index. The product was stored at $21^{\circ} \mathrm{C}, 35^{\circ} \mathrm{C}$ and $45^{\circ} \mathrm{C}$ for $210 \mathrm{~d}, 90 \mathrm{~d}$ and $42 \mathrm{~d}$. The product was sampled a least six times for each temperature and the results were used to define the kinetics for this deterioration. The order obtained for this kinetic reaction was cero and the constants found were $(0,31,0,173$ and 0,365$) \mathrm{meqO} / 2 \mathrm{~kg} \cdot \mathrm{d}$, in the same temperature order. Making use of the Arrhenius Model the calculated activation energy was $80960 \mathrm{~J} / \mathrm{mol}$, falling within the lipid oxidation rate. Finally, the expression for estimating the mayonnaise shelf life is described by the equation Shelf life $=10^{(2,907-0,036 \cdot \mathrm{T})}$.
\end{abstract}

Key words: mayonnaise, shelf life, kinetic, lipid oxidation rate.

Recibido: 29 de mayo del 2008 • Aprobado: 16 de septiembre del 2008

\section{INTRODUCCIÓN}

Un alimento es un sistema fisicoquímico y biológico activo, por lo que la calidad del mismo es un estado dinámico que se mueve hacia niveles más bajos respecto al tiempo. Existe un tiempo determinado, después de haber sido producido, en que el producto mantiene un nivel requerido de sus propiedades sensoriales y de seguridad, bajo ciertas condiciones de almacenamiento. Este constituye el período de vida útil o de anaquel del alimento (Casp, 1999; Kuntz, 1991). Por ello, en la industria de alimentos es importante determinar la vida útil de los productos para suplirlos y mantenerlos en el mercado sin ningún problema de seguridad o de rechazo por los consumidores.

La vida útil de un producto depende de factores ambientales, de la humedad, de la temperatura de exposición, del proceso térmico al que se somete y de la calidad de las materias primas, entre otros. El efecto de estos factores se manifiesta como el cambio en las cualidades del alimento que evitan su venta: cambios de sabor, color, textura o pérdida de nutrientes (Potter, 1978). Kuntz (1991) se refiere a que el final de la vida útil de un producto se alcanza cuando ya no mantiene las cualidades requeridas para que el consumidor final lo utilice. 
Para determinar la vida útil de un alimento o producto, primero deben identificarse las reacciones químicas o biológicas que influyen en la calidad y seguridad del mismo, considerando la composición del alimento y el proceso a que es sometido y se procede a establecer las reacciones más críticas en la calidad (Casp, 1999; Rondon, Pacheco y Ortega, 2004).

El tiempo de vida útil se puede estimar mediante varios métodos: pueden tomarse valores reportados en la literatura especializada de alimentos similares y bajo condiciones similares al producto de nuestro interés; se pueden monitorear las quejas de los consumidores para orientar los posibles valores de vida útil; se pueden evaluar atributos de calidad del alimento que varían durante la vida útil en anaquel o mediante pruebas aceleradas (CITA, 2005).

Los ensayos en anaquel ofrecen excelentes datos, pero presentan, en algunos casos, el inconveniente del tiempo prolongado para su adquisición. Entre las consecuencias están que el dato obtenido es puntual y se obtiene en un lapso que puede no ser práctico para la empresa, como en el caso del lanzamiento de nuevos productos.

Para las pruebas de vida útil acelerada se deben tomar en cuenta no solamente la selección de las temperaturas para realizar las pruebas, sino que debe establecerse el diseño estadístico experimental, realizar las respectivas mediciones por duplicado o triplicado para evaluar las desviaciones de las muestras, y así, evaluar de manera más apropiada la vida útil. Esto sin dejar de lado el hecho de que existe siempre un error asociado con la naturaleza del sistema biológico que generalmente es complejo.

Para los estudios acelerados se debe planear cuidadosamente el diseño experimental que contemple las variables por evaluar y controlar las variables que no se desean evaluar para evitar que interfieran en las mediciones, y por ende, en los resultados. Es esencial establecer aquellas variables críticas y disponer de métodos de cuantificación de repuesta rápida, pero confiable (Labuza, 1985).

Los estudios de vida útil acelerados, consisten en incubar el alimento bajo condiciones controladas y a diferentes temperaturas. Estas temperaturas deben ser mayores a las de almacenamiento y las de comercialización para permitir que las reacciones de deterioro se aceleren y se obtengan valores en períodos más cortos (Rodríguez, 2004).

Las pruebas de laboratorio simulan las condiciones reales, pero existen variables como las condiciones de transporte, cambios de presión, fluctuaciones de temperatura, entre otras, que son difíciles de duplicar. Por lo tanto, los resultados obtenidos son estimaciones de la vida útil del alimento. Cuando se utiliza un panel de expertos, es importante disponer de una muestra control o patrón del producto que se mantiene a temperaturas menores que las de almacenamiento y las de comercialización, para retardar las reacciones de deterioro.

Dado que existe poca información respecto a su vida útil y la energía de activación, este trabajo tiene por objetivo el estimar la vida útil de una mayonesa natural mediante pruebas aceleradas.

\subsection{Modelo para la degradación cinética}

Se ha encontrado que el deterioro de los alimentos sigue modelos de orden cero o primer orden; en alimentos con un alto contenido de grasa o lípidos predominan las reacciones de oxidación y estas siguen un comportamiento de orden cero (Labuza, 1984; Labuza, 1985; Pozo, 1992; Casp, 1999). El modelo para la reacción de orden cero se presenta en la ecuación (1).

$-\frac{\mathrm{dX}}{\mathrm{dt}}=\mathrm{k}$

Integrando la ecuación (1) y reacomodando, se tiene la ecuación de una línea recta con pendiente k; siendo k la constante específica de reacción y cuyo valor depende de la temperatura.

$\mathrm{X}_{\mathrm{f}}=\mathrm{X}_{0}-\mathrm{kt}_{\mathrm{u}}$

Con $\mathrm{X}_{0}$ como la intersección con el eje $\mathrm{Y}$. 


\subsection{Dependencia de la temperatura}

Puesto que la constante de velocidad de reacción es función de la temperatura, esta dependencia es descrita por la ecuación de Arrhenius. El modelo de Arrhenius describe la relación de la constante de velocidad de reacción con la temperatura según la ecuación (3).

$\mathrm{k}=\mathrm{Ae}^{\left(-\mathrm{E}_{\mathrm{a}} / \mathrm{RT}\right)}$

Al aplicar logaritmos a ambos lados de la ecuación (3) se obtiene la ecuación de una línea recta con pendiente $\mathrm{E}_{\mathrm{a}} / \mathrm{R}$, tal como se expresa en la ecuación (4); el término $\mathrm{E}_{\mathrm{a}}$ puede evaluarse para conocer el valor de la energía de activación.

$\ln \mathrm{k}=\ln \mathrm{A}-\frac{\mathrm{E}_{\mathrm{a}}}{\mathrm{R}} \cdot \frac{1}{\mathrm{~T}}$

Donde:

k constante de velocidad de reacción.

A factor de frecuencia.

$\mathrm{E}_{\mathrm{a}}$ energía de activación.

$\mathrm{R}$ constante de los gases ideales.

$\mathrm{T}$ temperatura absoluta (K).

\section{METODOLOGÍA Y EQUIPO EXPERIMENTAL}

\subsection{Características de la mayonesa}

Los componentes principales de la mayonesa natural son: aceite, agua, almidón y huevo. De ellos, el aceite es el ingrediente que está en mayor cantidad, razón por la cual se considera este componente para realizar el seguimiento a la degradación de la mayonesa, dado que el deterioro de este producto en mayor medida es originado por reacciones de oxidación, que producen la rancidez.

\subsection{Muestras}

Se toman muestras del mismo lote de producción para el estudio. Se almacenan en refrigeración a $5^{\circ} \mathrm{C}$ para mantenerlas "frescas" hasta su colocación en la incubadora, para iniciar el estudio de vida útil a una temperatura mayor.

\subsection{Empaque del producto}

El empaque del producto utilizado es flexible y su estructura está compuesta de poliéster, tintas, adhesivos y polietileno. El poliéster actúa como una barrera contra la humedad, posee alta resistencia a la ruptura y a las altas temperaturas. Las tintas funcionan como barreras contra la luz. El adhesivo tiene la función de unir las capas de los demás materiales y, si está en contacto con el alimento, en las uniones y en los bordes de los laminados, debe estar presente en mínima cantidad. El polietileno es flexible y se estira fácilmente, es una barrera contra el vapor de agua, no tiene olor ni sabor y es fácil de sellar mediante calor.

\subsection{Variable de respuesta}

Se estableció utilizar el índice de peróxidos como variable de respuesta, debido a que este se encuentra directamente relacionado con el sabor rancio que se percibe en las mayonesas y productos semejantes con altos contenidos de grasas.

\subsection{Metodología y procedimiento experimental}

El primer paso consiste en separar el aceite que contiene la mayonesa del resto de los ingredientes. Para lograr esto se toma como base la norma AOAC 983,23. Se pesan aproximadamente $15 \mathrm{~g}$ de mayonesa en un erlenmeyer de $500 \mathrm{~cm}^{3}$. Se le agregan $80 \mathrm{~cm}^{3}$ de metanol y $40 \mathrm{~cm}^{3}$ de cloroformo. Se coloca una pastilla magnética dentro del erlenmeyer y este se pone en baño maría sobre una plancha magnética 
a una temperatura entre $45^{\circ} \mathrm{C}$ y $50^{\circ} \mathrm{C}$. Se deja en agitación durante $15 \mathrm{~min}$. Se le agregan $40 \mathrm{~cm}^{3}$ más de cloroformo y se mezclan durante otros 5 min, y luego se agregan $40 \mathrm{~cm}^{3}$ de agua destilada y se mezcla 1 min más. Se deja reposar para que se separe la fase acuosa de la oleosa. Utilizando una pipeta se transfiere la fase oleosa a tubos de ensayo. Se ponen los tubos a centrifugar durante $10 \mathrm{~min}$ a $3000 \mathrm{rpm}$. Se pipetean aproximadamente $65 \mathrm{~cm}^{3}$ de la mezcla aceite-cloroformo en un erlenmeyer de $250 \mathrm{~cm}^{3}$ y se coloca en baño maría para evaporar el cloroformo.

Para determinar el índice de peróxidos se utiliza como base el método Standard Internacional ISO 3960 , la metodología seguida por Rondon et al (2004) y los ajustes al procedimiento establecidos por García (2008). El aceite obtenido de la separación de la mayonesa se pesa y se agregan 50 $\mathrm{cm}^{3}$ de una solución de ácido acético-cloroformo y se agita para mezclar. Se añaden $0,5 \mathrm{~cm}^{3}$ de una solución saturada de yoduro de potasio, se dejan reaccionar durante $1 \mathrm{~min} \pm 1 \mathrm{~s}$ y se agregan $0,5 \mathrm{~cm}^{3}$ de solución de almidón. Se adicionan 30 $\mathrm{cm}^{3}$ de agua destilada inmediatamente después. Se inicia la titulación con una solución de tiosulfato de sodio $0,06 \mathrm{M}$, de forma gradual y constante, hasta que el color azulado desaparezca.

Se prepara un blanco siguiendo el mismo procedimiento, pero sin muestra. Las determinaciones se realizan por duplicado. El consumo de reactivo fue de $0 \mathrm{~cm}^{3}$.

Las unidades de medición del índice de peróxidos utilizadas son miliequivalentes de oxígeno activo por kilogramo de aceite, $\mathrm{meqO}_{2} / \mathrm{kg}$. Para el cálculo del índice de peróxido se utiliza la siguiente ecuación ISO 3960.
$\mathrm{IP}=\frac{1000 \cdot\left(\mathrm{V}-\mathrm{V}_{0}\right) \cdot \mathrm{c}}{\mathrm{m}}$

\section{Donde:}

IP índice de peróxido variable de respuesta, en meqO $\mathrm{O}_{2} / \mathrm{kg}$.

$\mathrm{V}$ volumen de solución de tiosulfato de sodio utilizado para la determinación, en $\mathrm{cm}^{3}$.

$\mathrm{V}_{0}$ volumen de solución de tiosulfato de sodio utilizado en el blanco, en $\mathrm{cm}^{3}$.

c concentración de la solución de tiosulfato, en $\mathrm{mol} / \mathrm{L}$.

m masa de la muestra, en $\mathrm{g}$.

\subsection{Determinación de la periodicidad de análisis}

Labuza (1985) y Neter (1983) indican que la cantidad mínima de temperaturas para conducir un estudio de vida útil son tres. Para este estudio se establecieron las temperaturas de $21{ }^{\circ} \mathrm{C}, 35{ }^{\circ} \mathrm{C}$ y de $45{ }^{\circ} \mathrm{C}$. Las temperaturas de $35{ }^{\circ} \mathrm{C}$ y $45{ }^{\circ} \mathrm{C}$ se escogieron para establecer una diferencia de $10{ }^{\circ} \mathrm{C}$ y poder calcular el valor de $\mathrm{Q}_{10}$ que representa la razón de las constantes de velocidad de reacción a las temperaturas mencionadas. En el Cuadro 1 se presenta la periodicidad de la toma de muestras de mayonesa; el muestreo para la temperatura de $21^{\circ} \mathrm{C}$ se extendió a siete meses y para las temperaturas de $35{ }^{\circ} \mathrm{C}$ y $45^{\circ} \mathrm{C}$, se extendió a tres meses y cuarenta y dos días, respectivamente.

Cuadro 1. Secuencia de muestreo para el análisis de la mayonesa.

Temperatura de
almacenamiento

$\left({ }^{\circ} \mathrm{C}\right)$

21

35

45

Período
medición

(d)

30

15

7

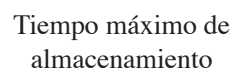

Tiempo máximo de almacenamiento

(d)

180

90

42
Muestreo

(d)

$30,60,90,120,150,180,210$

$15,30,45,60,75,90$

$7,14,21,28,35,42$ 


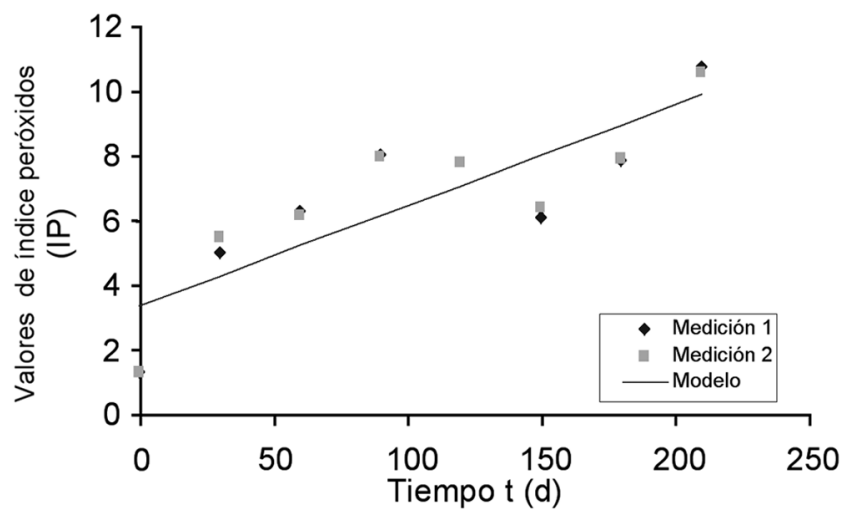

Figura 1. Índice de peróxidos con su repetición en función del tiempo a una temperatura de $21^{\circ} \mathrm{C}$. Fuente: (La autora y el autor).

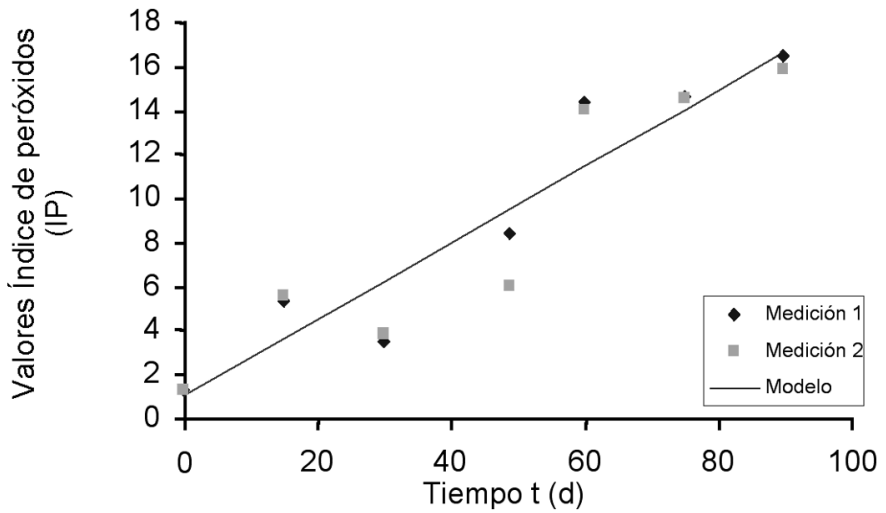

Figura 2. Índice de peróxidos con su repetición en función del tiempo a una temperatura de $35^{\circ} \mathrm{C}$.

Fuente: (La autora y el autor).

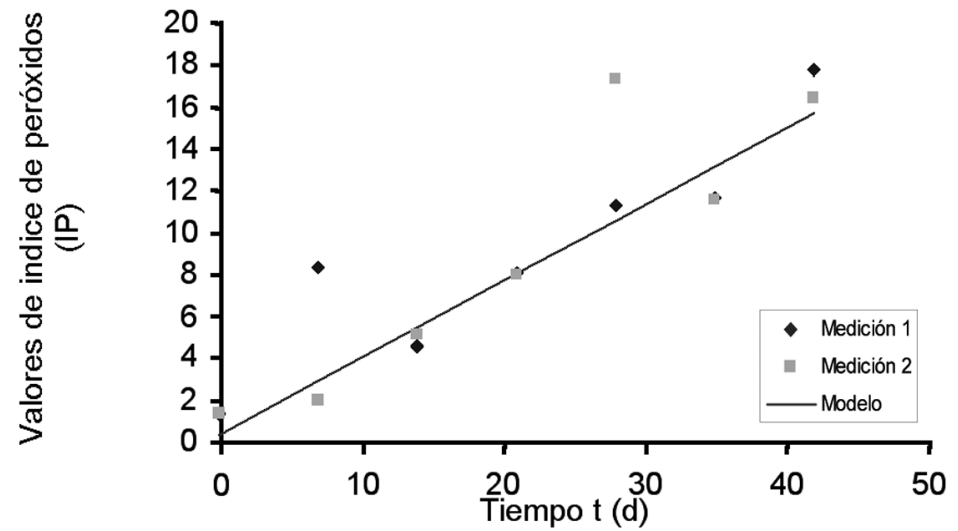

Figura 3. Índice de peróxidos con su repetición en función del tiempo a una temperatura de $45^{\circ} \mathrm{C}$.

Fuente: (La autora y el autor). 


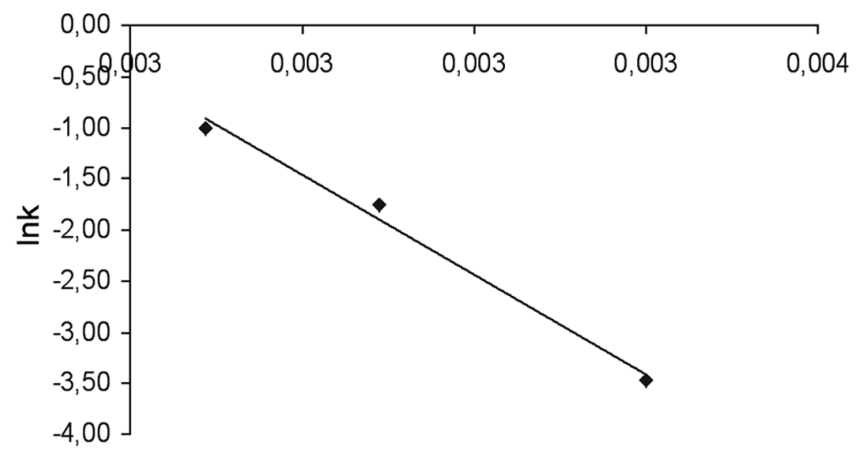

$1 / \pi(1 / K)$

Figura 4. Gráfico del lnk en función de 1/T.

Fuente: (La autora y el autor).

\subsection{Equipo experimental}

Incubadora: Lab Line Imperial III-310 que permite establecer las temperaturas de interés: $\left(21{ }^{\circ} \mathrm{C}, 35{ }^{\circ} \mathrm{C}\right.$ y $\left.45{ }^{\circ} \mathrm{C}\right) \pm 1{ }^{\circ} \mathrm{C}$; cuenta con el espacio suficiente para colocar las muestras de mayonesa que deben tomarse periódicamente para el análisis de peróxidos.

Centrífuga: International Equipment Company HN-SII, para centrifugar las muestras durante los procesos de extracción con solventes, a velocidades de $3000 \mathrm{rpm}$.

\section{DISCUSIÓN DE RESULTADOS}

Los resultados del índice de peróxidos en función del tiempo para cada temperatura se muestran en las Figuras 1, 2 y 3.

Se puede observar en las Figuras 1, 2 y 3 que el índice de peróxidos en la mayonesa aumenta respecto al tiempo y su comportamiento es lineal. En la Figura 3 se observan dos puntos alejados de dicha tendencia, pero que fueron excluidos después de su análisis estadístico, para la determinación de las ecuaciones cinéticas correspondientes. Las regresiones lineales obtenidas de estas figuras se presentan en las ecuaciones (6), (7) y (8).

$\mathrm{IP}=3,331+0,31 \cdot \mathrm{t}$

$\mathrm{IP}=0,994+0,173 \cdot \mathrm{t}$
$\mathrm{IP}=0,281+0,365 \cdot \mathrm{t}$

Con las tres constantes obtenidas, representadas por las pendientes de las ecuaciones (6), (7) y (8), para las tres temperaturas estudiadas, se aplicó el modelo de Arrhenius, en la forma como se expresa en la ecuación (4) (figura del ln k en función de 1/T).

La ecuación que se obtiene de esta regresión lineal es la ecuación (9).

$\ln \mathrm{k}=-9737,6 \cdot \frac{1}{\mathrm{~T}}+29,693$

El valor de la energía de activación que se calcula de esta ecuación es $80960 \mathrm{~J} / \mathrm{mol}$. Torres, Guerra y Rosquete, (2001) indican que la energía de activación para reacciones de oxidación de lípidos se encuentra en el ámbito de $41842 \mathrm{~J} /$ mol a $104605 \mathrm{~J} / \mathrm{mol}$. El resultado de este estudio se encuentra dentro de dicho ámbito.

Según las evaluaciones de paneles sensoriales efectuadas con jueces entrenados, se obtuvo un valor de índice de peróxidos de $7,96 \mathrm{meqO}_{2} / \mathrm{kg}$ para el cual dichos jueces consideran que el producto ya no reúne las cualidades necesarias para el consumo. Con este valor y las ecuaciones (6), (7) y (8) se estimaron los valores puntuales de vida útil de la mayonesa, para las temperaturas de almacenamiento de $(21,35$ y 45$){ }^{\circ} \mathrm{C}$. 


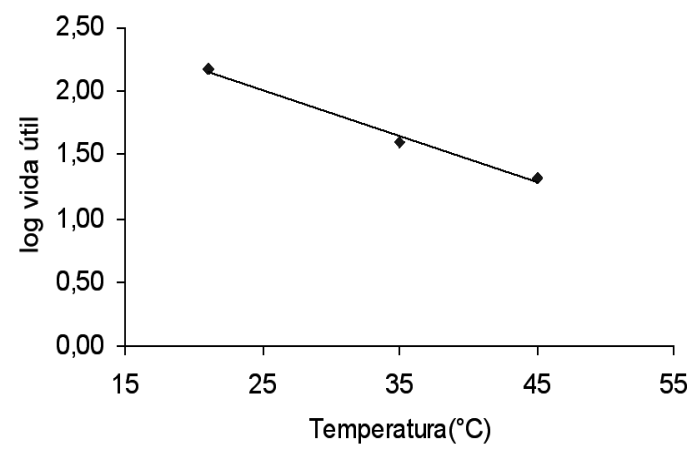

Figura 5. Gráfico del log de vida útil en función de las temperaturas.

Fuente: (La autora y el autor).

Se graficó el logaritmo de la vida útil a (21, 35 y 45$)^{\circ} \mathrm{C}$. La pseudo-transformación resultante se puede observar en la Figura 5, la que se puede utilizar siempre y cuando los ámbitos de temperatura sean estrechos (Labuza, 1984).

De la Figura 5 se obtiene mediante regresión lineal la ecuación (10) con la que puede estimarse la vida útil de la mayonesa para diferentes temperaturas de almacenamiento.

Log vida útil $=2,907-0,036 \cdot \mathrm{T}$

Y despejando la ecuación (10) se obtiene:

Vida útil $=10^{(2,907-0,036 \cdot \mathrm{T})}$

Donde $T$ está en ${ }^{\circ} \mathrm{C}$.

Cuadro 2. Valores de vida útil y $\mathrm{Q}_{10}$

$\begin{array}{ccc}\begin{array}{c}\text { Temperatura } \\ \left({ }^{\circ} \mathrm{C}\right)\end{array} & \begin{array}{c}\text { Vida útil } \\ (\mathrm{d})\end{array} & \mathrm{Q}_{10} \\ 21 & 149 & 2,97 \\ 35 & 40 & 2,70 \\ 45 & 21 & 2,54\end{array}$

Fuente: (La autora y el autor).
En el Cuadro 2 se presentan los valores estimados de vida útil y los valores de $\mathrm{Q}_{10}$.

\section{CONCLUSIONES}

1. De acuerdo con las ecuaciones obtenidas para el aumento del índice de peróxidos en función del tiempo, el orden de reacción para la oxidación del aceite de la mayonesa es cero y la energía de activación es de $81 \mathrm{~kJ} / \mathrm{mol}$.

2. La vida útil estimada del producto a $21^{\circ} \mathrm{C}$, $35{ }^{\circ} \mathrm{C}$ y $45^{\circ} \mathrm{C}$ es de $149 \mathrm{~d}, 40$ d y $21 \mathrm{~d}$, respectivamente.

3. Con estos tiempos y las temperaturas estudiadas, se obtuvo la ecuación general para estimar la vida útil de este producto, para diferentes temperaturas de almacenamiento, la cual está descrita por la ecuación: Vida útil $=10^{(2,907-0,036-\mathrm{T})}$.

4. La vida útil predicha a una temperatura alta es corta, razón por la que empresas que manufacturen productos de mayonesa y las coloquen en regiones donde prevalecen temperaturas ambientales altas, deben contar con un programa de rotación del producto.

\section{REFERENCIAS BIBLIOGRÁFICAS}

Casp, A. \& April, J. (1999). Procesos de conservación de alimentos. Madrid: Ediciones Mundi-Prensa.

Centro Nacional de Ciencia y Tecnología de Alimentos (CITA). (2005). Curso teórico práctico: Vida útil de alimentos aplicados a la industria. San José Costa Rica.

García, C. (2008). Estimación de la vida útil de un producto alimenticio pasteurizado y uno acidificado mediante pruebas aceleradas. Proyecto de graduación para optar por el grado de Licenciatura en Ingeniería Química, Universidad de Costa Rica, San José, Costa Rica.

International Organization for Standardization (ISO). (2001). Animal and vegetable fats and oils-Determination of peroxide value ISO 3960 ( $3^{\mathrm{a}}$ ed.).

Kuntz, L. (1991). Accelerated shelf life testing. Nueva York: Weeks Publishing Co. 
Labuza, T. (1984). Aplication of chemical kinetics to deterioration of foods. Chemical Education, 61(4), 348-358.

Labuza, T. \& Schmidt, M. (1985). Accelerated shelf-life dating of foods. Food Technology, 39 (9), 57-134.

Neter, J. (1983). Applied linear regression models. Illinois: Richard D. Irwin, Inc.

Association of Official Analytical Chemists. (2002). Official methods of analysis of the Association of Official Analytical Chemists 983.23. Fats in Foods.

Potter, N. (1978). La ciencia de los alimentos $\left(2^{\mathrm{a}}\right.$ ed.). México D. F: Edutex, S. A.

Pozo, R. M. (1992). Proceso de la rancidez oxidativa de los aceites y grasas. Metodología, Anales de la Real Academia de Farmacia (58), 17-29.

Rodríguez, V. (2004). Estimación de la vida útil de la harina de pejibaye, obtenida por deshidratación. Tesis para optar por el grado de Licenciatura en Tecnología de Alimentos, Universidad de Costa Rica, San José, Costa Rica.

Rondon, E., Pacheco, E. \& Ortega, F. (2004). Estimación de la vida útil de un análogo comercial de mayonesa utilizando el factor de aceleración Q10. Facultad de Agronomía, Universidad Central de Venezuela, 4(21), 68-83.
Torres, A., Guerra, M. \& Rosquete, Y. (2001). Estimación de la vida útil de una fórmula dietética en función de la disminución de lisina disponible. Ciencia y Tecnología de Alimentos, Brasil, 21(2).

\section{SOBRE EL AUTOR Y LA AUTORA}

\section{Claudia García Baldizón}

Licenciada en Ingeniería Química de la Universidad de Costa Rica

Coordinadora de Evaluación Sensorial Centroamérica

Departamento de Investigación y Desarrollo

Empresa Unilever de Centroamérica

Teléfono: (506) 22258650

Facsímil: (506) 22812074

Correo electrónico: cgarcia_baldizon@yahoo.com

\section{Manuel E. Molina Córdoba}

Licenciado en Ingeniería Química de la Universidad de Costa Rica

Docente e Investigador de la Facultad de Ingeniería Escuela de Ingeniería Química

Teléfono: (506) 22074620 / (506) 22853486

Facsímil: (506) 22255622

Correo electrónico: emolina@fing.ucr.ac.cr 